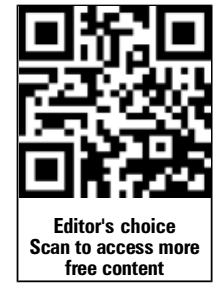

${ }^{1} J o h n$ Hunter Clinic for Sexual Health, Chelsea and Westminster Hospital NHS Foundation Trust, London, UK ${ }^{2}$ Department of HIV and STIs, Health Protection Agency, London, UK

${ }^{3}$ University of Liverpool, Liverpool, UK

\section{Correspondence to}

Dr Ann Kathleen Sullivan, John Hunter Clinic for Sexual Health, Chelsea and Westminster Hospital NHS Foundation Trust, 369 Fulham Road, London SW10 9NH, UK;

Ann.Sullivan@Chelwest.nhs.uk

Received 17 September 2012 Revised 17 September 2012 Accepted 26 November 2012

\title{
Non-disclosure of HIV status in UK sexual health clinics - a pilot study to identify non-disclosure within a national unlinked anonymous seroprevalence survey
}

\author{
Ann Kathleen Sullivan, ${ }^{1}$ Emma J Savage, ${ }^{2}$ Catherine M Lowndes, ${ }^{2}$ George Paul, ${ }^{1}$ \\ Gary Murphy, ${ }^{2}$ Simon Carne, ${ }^{2}$ David J Back, ${ }^{3} 0$ Noel Gill ${ }^{2}$
}

\section{ABSTRACT}

Objectives To identify if HIV-infected individuals attending genitourinary clinics in the UK are not disclosing their HIV status, and to examine the potential utility of drug detection as a method to indicate nondisclosure.

Methods HIV-positive samples from the unlinked anonymous seroprevalence survey from one London centre in 2009 had viral load (VL) assays performed to identify samples with $\mathrm{VL}$ below the level of detection (50 copies/ml, VLBLD) or $<1000$ copies/ml. After data matching, known HIV positives were excluded and the remaining samples analysed for the presence of a panel of antiretroviral drugs.

Results Of 130 HIV-positive samples with sufficient clinical information and not undergoing an HIV test, 18 were classified as remaining undiagnosed after the clinic visit. Thirteen (72\%, 95\% Cl: $47 \%$ to $90 \%$ ) had a VLBLD $(n=11)$ or $\mathrm{VL}<1000$ copies/ml $(n=2)$. Eight had sufficient volume to undergo ARV testing, and all were positive for the presence of drug; all with therapeutic levels of clinically appropriate combinations.

Conclusions Non-disclosure of HIV status occurs among individuals attending sexual health services in the UK. This study demonstrates the feasibility and utility of using both VL and ARV assays in serum samples. Furthermore, the close correlation of detection of ARV with VLBLD suggests drug detection would be a useful tool to monitor non-disclosure prospectively, thus enabling the use of stored serum samples in future studies. The extent to which these findings can be extrapolated to other settings, and the potential impact of non-disclosure on undiagnosed estimates warrants urgent prospective study.

\section{INTRODUCTION}

Currently, of all people living with HIV in the UK, it is estimated that $24 \%$, approximately 22200 individuals, are unaware of their infection. ${ }^{1}$ This figure is derived from statistical modelling of multiple surveillance- and survey-based data sources, One of the surveillance programmes is the unlinked anonymous seroprevalence survey: GUMAnon Survey, ${ }^{2}$ conducted at 16 UK genitourinary (GU) clinics. The methodology is described elsewhere; ${ }^{2}$ in brief, unlinked residue from routine syphilis blood specimens are tested for HIV antibodies; broad-risk categories and whether the individual is categorised as previously diagnosed, diagnosed at the clinic visit or left the clinic unaware of their HIV status are available. Using 2009 data, it was estimated that $27 \%$ of previously undiagnosed individuals left the clinic unaware of their positive HIV status. Anecdotally, expert opinion has been that some patients aware of their positive HIV status attend GU services but do not disclose their status. A report by Whitlock and colleagues, ${ }^{3}$ describes a small (1.9\%) proportion of patients self-reporting this behaviour. This phenomenon could result in an overestimation of the proportion of individuals who are unaware of their status. We therefore undertook the following study to objectively determine if this behaviour occurs, and to examine the potential utility of drug detection to indicate non-disclosure.

Several assumptions were made underpinning the methodology and interpretation of the data. Given that London HIV treatment rates among individuals accessing HIV care are over $80 \%$, and the effectiveness of antiretroviral (ARV) therapy in clinical practice exceeds $90 \%,{ }^{1}$ if non-disclosure behaviour was prevalent, there would be a significant number of non-disclosers (ND) on ARV therapy, and a significant number of these individuals (ND on ARV) would have undetectable or very low viral loads (VL). Furthermore, of all diagnosed HIV patients, only a very small proportion $(0.15 \%)$ are elite controllers with undetectable VLs when not on treatment. ${ }^{4}$

\section{METHODS}

All HIV-positive samples identified through GUMAnon from one London centre in 2009 were included. VL assays identified samples with VL below the level of detection (50 copies/ml, VLBLD) or $<1000$ copies/ml and, after matching with clinical information to exclude samples from those previously diagnosed, these samples were analysed for the presence of a panel of ARV drug.

\section{HIV antibody test}

Pooled specimens were screened for HIV antibodies using enzyme immunoassay (Abbott Diagnostics, Maidenhead). Reactive pools were retested singularly, and reactive specimens subsequently tested with an in-house IgG class specific capture assay. ${ }^{5}$ Discrepant results were tested by an in-house IgG 
antibody capture enzyme-linked immunosorbent assay (GACELISA) and, where necessary, by an immunocomb or western blot assay.

\section{VL assay}

VL assays were performed using a real-time quantitative Taqman probe-based assay multiplexed with an internal control using a series of quantified HIV-1 standards to determine virus concentration.

\section{Drug assays}

Samples were analysed using validated, high-performance liquid chromatography mass spectrometry. ${ }^{6} 7$ Three assays were performed to detect saquinavir ritonavir, amprenavir, lopinavir, atazanavir, darunavir, nevirapine, etravirine, maraviroc, raltegravir (assay one); lamivudine, emtricitabine, tenofovir (assay two) and efavirenz (assay three).

\section{Ethics}

Ethical approval was not required for this public health surveillance programme; NRES guidance (http://www.nres.npsa.nhs. uk/applications/is-your-project-research/), Department of Health statement 23rd November 1988 (Gill et al, BMJ 1989;299:1295-98), confirmed by National Research Ethics Service, National Patient Safety Agency (March 2011) and in respect of residual, acellular material GAfREC (paragraph 2.3.11).

\section{RESULTS}

We identified 130 HIV-positive samples with matching clinical information. Of these, 28 were from patients not previously known to be HIV positive, of whom 10 were recorded in the GUMAnon survey as having had a diagnostic HIV test in clinic; the remaining 18 did not undergo diagnostic HIV testing, and were therefore classified as remaining undiagnosed after the clinic visit. Thirteen of these $(72 \%, 95 \% \mathrm{CI}$ : $47 \%$ to $90 \%$ ) had a VLBLD $(n=11)$ or a VL $<1000$ copies/ml $(n=2)$. Eight of these samples (four men who have sex with men, one heterosexual, three unknown) had sufficient volume to undergo ARV testing, and all were positive for the presence of drug. All drug-positive samples had therapeutic levels of clinically appropriate combinations: four had lamivudine or emtricitabine and tenofovir with efavirenz, nevirapine or boosted darunavir detected; three had lamivudine or emtricitabine and efavirenz or boosted darunavir and one had boosted lopinavir.

\section{DISCUSSION}

This is the first published objective evidence that nondisclosure of HIV status as a phenomenon exists in patients attending GU clinics in the UK. We sought to explore the utility of various laboratory tests to identify it, and this study demonstrates the feasibility and utility of using both VL assays and assays for ARVs in serum samples. This study, performed in one London clinic, revealed significant proportions (72\%) of samples from individuals classified as HIV seropositive but unaware of their status to have an undetectable or very low $\mathrm{VL}$, suggestive of being on ARV therapy. This was verified through subsequent detection of ARVs in the stored serum samples. The VL and ARV assay results correlate extremely well -all samples with a low or undetectable VL that were tested for ARVs were positive. A strength of the study is the compatibility of the detected ARV regimens and the adequate levels measured, making false positive results unlikely. Furthermore, the close correlation of detection of ARV with VLBLD suggests drug detection would be a useful tool to monitor nondisclosure prospectively, thus enabling the use of stored serum samples in future studies.

These results indicate that non-disclosure of HIV occurs among individuals attending a GU clinic in central London. Due to the small sample size and potential selection bias it is not possible to generalise these results. Given the high proportion of individuals classified within this study as NDs, the extent to which these finding can be extrapolated to other clinics, and the degree to which they may influence estimates of the proportion of undiagnosed HIV in the community warrant further study. This is because in England, most of the estimate of undiagnosed HIV infection in the population of clinic attendees is based on the prevalence of undiagnosed HIV infection on arrival at the clinic, and non-disclosure affects the estimate of the prevalence of HIV infection in attendees upon leaving a clinic. However non-disclosure is likely to influence it to a degree and, therefore, prospective objective measurements of non-disclosure should be considered in surveillance programmes which estimate rates of undiagnosed HIV in all countries undertaking such work.

Acknowledgements The authors would like to acknowledge the contribution by the clinical and laboratory staff involved in GUMAnon and at Liverpool University.

Contributors AKS had the original concept, designed the pilot study, coordinated GUMAnon clinically, analysed the data and wrote the initial draft of the paper. EJS coordinated GUMAnon sample analysis, analysed the data and contributed to writing the initial draft. CML analysed the data and revised the manuscript. GP coordinated GUMAnon clinically and commented on the final draft. GM and SC analysed GUMAnon samples and contributed to writing the initial draft. DJB performed drug analysis, analysed the data and contributed to writing the initial draft. ONG analysed data and revised the manuscript. EJS CML and ONG coordinate and run GUMAnon nationally

Funding None.

Competing interests None.

Provenance and peer review Not commissioned; externally peer reviewed.

\section{REFERENCES}

1. Health Protection Agency. HIV in the United Kingdom; 2011 Report. http://www. hpa.org.uk/webc/HPAwebFile/HPAweb C/1317131685847 (accessed 20 Aug 2012).

2. Health Protection Agency. Unlinked Anonymous Survey of Genitourinary Clinic Attendees (GUM Anon Survey). http://www.hpa.org.uk/web/ HPAweb\&HPAwebStandard/HPAweb_C/1202115 (accessed 20 Aug 2012).

3. Whitlock GG, Lowndes CM, Mercey DE, et al. Do HIV-infected individuals test for sexually transmitted infections at another sexual health clinic? Sex Transm Infect 2011;87:253.

4. Grabar S, Selinger-Leneman H, Abgrall S, et al. Prevalence and comparative characteristics of long-term nonprogressors and HIV controller patients in the French Hospital Database on HIV. AIDS 2009;23:1163-9.

5. Parry JV, Connell JA, Reinbott P, et al. GACPAT HIV $1+2$ : a simple, inexpensive assay to screen for, and discriminate between, anti-HIV 1 and anti-HIV 2. J Med Virol 1995;45:10-16.

6. Else LJ. Validation of a rapid and sensitive high-performance liquid chromatography-tandem mass spectrometry (HPLC-MS/MS) assay for the simultaneous determination of existing and new antiretroviral compounds. $J$ Chromatogr B Analyt Technol Biomed Life Sci 2010;878:1455-65.

7. Else LJ, Jackson A, Puls $R$, et al. Pharmacokinetics of lamivudine and lamivudine-triphosphate after administration of 300 milligrams and 150 milligrams once daily to healthy volunteers: results of the ENCORE 2 study. Antimicrob Agents Chemother 2012;56:1427-33. 